Congreso Internacional de Gestión Educativa, 06 y 07 de noviembre de 2020.

Pontificia Universidad Católica del Perú

\title{
El rol del director, ¿cómo lograr el aprendizaje de los estudiantes?
}

\author{
Gamarra Ramos, Ana Celia \\ agamarra_ramos@hotmail.com \\ https://orcid.org/0000-0002-3124-6738
}

Resumen: La experiencia en gestión escolar se desarrolla en el contexto de pandemia mundial a causa del COVID-19, en el cual las escuelas se cierran por la emergencia sanitaria y el aislamiento social obligatorio para evitar la propagación del virus. En este contexto atípico para la educación peruana era necesario e imperante reinventar el rol del director para repensar y reformular acciones que permitieran asegurar que todos los estudiantes accedan a las clases a distancia a través de la estrategia "Aprendo en Casa" formulada y ejecutada por el Ministerio de Educación del Perú. En este espacio de educación a distancia, era necesario reimaginar la gestión escolar para asegurar que los estudiantes logren aprendizajes; utilizando para ello, diversas estrategias para atender y asegurar una atención de calidad haciendo uso de todos los recursos humanos, materiales y tecnológicos disponibles en la institución y asegurar, por un lado, la participación activa de los estudiantes en las clases a distancia y que se desarrolle todas las competencias del Currículo Nacional de la Educación Básica para asegurar el logro de los aprendizajes de los estudiantes.

Palabras clave: Gestión escolar; logros de aprendizaje

\section{The role of the director, how to achieve student learning?}

Abstract: The experience in school management is developed in the context of the global pandemic caused by COVID-19, in which schools are closed due to the health emergency and mandatory social isolation to prevent the spread of the virus. In this atypical context for Peruvian education, it was necessary and imperative to reinvent the role of the director to rethink and reformulate actions that would ensure that all students access distance classes through the strategy "I learn at home" formulated and executed by the Ministry of Education of Peru. In this distance education space, it was necessary to reimagine school management to ensure that students achieve learning; using for this, various strategies to attend and ensure quality care making use of all human, material and technological resources available at the institution and ensure, on the one hand, the active participation of students in distance classes and that develop all the competencies of the National Curriculum of Basic Education to ensure the achievement of student learning.

Keywords: School Management; learning achievements

\section{Introducción}

La actual situación de pandemia mundial, generado por el COVID-19, la declaratoria del estado de emergencia sanitaria, el aislamiento social obligatorio y aunado a ello, la crisis en la educación que ya se venía atravesando en el país por diversas causas, entre ellas, la existencia de brechas digitales, bajos logros de aprendizaje de los estudiantes marcado por una abismal diferencia entre contextos rurales y urbanos, resultados nada 
Congreso Internacional de Gestión Educativa, 06 y 07 de noviembre de 2020.

Pontificia Universidad Católica del Perú

alentadores en las evaluaciones estandarizadas como Programme for International Student Assessment (PISA) y Evaluación Censal de estudiantes (ECE); ha generado repensar la gestión escolar en las instituciones educativas para garantizar el derecho a la educación de los estudiantes y que logren aprender en el marco de las clases a distancia a través de la estrategia promovida por el Ministerio de Educación "Aprendo en casa" (EAC).

Desde el mes de marzo del presente año, todas las instituciones educativas a nivel nacional interrumpen el desarrollo de las clases presenciales e inician con un nuevo reto educativo a partir del 06 de abril, con una estrategia nunca antes experimentada en el sector educación. El inicio de clases a distancia se apertura con una serie de situaciones inciertas tanto para los que dirigen el sector y los que conformamos el sistema educativo peruano.

Docentes y estudiantes que son parte importante de la dinámica educativa nos vimos de pronto, ante un hecho jamás vivido. Teniendo en cuenta que, la escuela es el lugar donde se crean oportunidades para que los estudiantes se involucren de manera activa en el proceso de enseñanza aprendizaje por lo que, la prioridad era que los docentes, promuevan en las aulas aprendizajes significativo (Talanquer, 2015). Ante este nuevo panorama, no existía otra alternativa que las aulas presenciales se conviertan en aulas a distancia y que este espacio enriquecedor, único y especial que generaba un vínculo importante entre docentes y estudiantes sea relegado a un trabajo a distancia de pronto frío y sin tener en cuenta las inmensas brechas digitales que existen entre contextos urbanos y rurales y las clases sociales existentes en el país.

Es así que, en este escenario de incertidumbre, de nuevos retos y demandas, de crisis de brecha digital; era necesario reinventar la gestión escolar y poner como prioridad que los estudiantes accedan a las clases a distancia y sobre todo asegurar que sigan aprendiendo. Para ello, se necesitaba otorgar una mirada esperanzadora a esta incertidumbre educativa. Era urgente que, la gestión escolar calzara con mucho tino y responsabilidad ante este reto atípico.

Por lo tanto, el rol del director como líder pedagógico, implicaba repensar la gestión educativa en este nuevo escenario y esto implicaba reorganizar y replantear la gestión escolar, con acciones inmediatas que involucraba la participación de todo el personal de la institución educativa para garantizar primero, la cobertura del 100\% de estudiantes a las clases a distancia, de definir los mecanismos más efectivos para que los estudiantes acedan a las clases a distancia.

De acuerdo a la base de datos institucional, al mes de abril del 2020 se inicia las clases con el $79 \%$ de estudiantes contactados; $5,4 \%$ de estudiantes no ubicados; $8,7 \%$ de estudiantes que no responden a las llamadas telefónicas; 93,5\% de estudiantes contaban con celular Smartphone y 6,5\% de estudiantes solo contaban con un celular convencional. De los cuales, el 52, 3\% cuenta con un celular propio y el 47,7\% utiliza celulares de sus padres y hermanos; y solo el $4 \%$ de estudiantes cuentan con celular con datos ilimitados; el 23\% de estudiantes cuentan con televisión en casa (Equipo tecnológico de la I.E.). Finalmente, para el mes de agosto, la data nos muestra que el $81 \%$ de estudiantes logran conectarse a la EAC a través de diversos medios de conectividad (TV, whatsapp, llamadas telefónicas); el $21 \%$ de estudiantes acceden a las clases a distancia con TV Nacional; el $46,5 \%$ a través de WhatsApp y un $6.1 \%$ de estudiantes trabajan con celular convencional y un $12,1 \%$ de estudiantes que no tienen contacto con la institución. 
Congreso Internacional de Gestión Educativa, 06 y 07 de noviembre de 2020.

Pontificia Universidad Católica del Perú

Por otro lado, el $66,7 \%$ de docentes y personal administrativo contaba con celular con datos ilimitados y el 33,3\% contaban con celulares con datos ilimitados y con deficiente cobertura para las clases a distancia y las reuniones colegiadas. Ante esta situación compleja, se movilizó todos los recursos humanos, materiales y tecnológicos con que cuenta la institución. Se sostuvo diálogos con los padres de familia, las autoridades locales para aunar esfuerzos en este proceso de las clases a distancia.

De lo dicho, era necesario que el rol trascendental del líder pedagógico implicaba marcar el camino para asumir con convicción y responsabilidad los retos y demandas que se presentaban para asegurar la cobertura de los estudiantes a las clases a distancia y garantizas el logro de aprendizajes.

\section{Referentes bibliográficos}

\section{Rol del directivo}

La crisis educativa actual, ha generado mayor organización e involucramiento a nivel institucional, reformular y redefinir estrategias, asimilar nuevos espacios educativos, enfrentar temores y angustias para asegurar la cobertura de los estudiantes a las clases a distancia y permitir que los estudiantes sigan aprendiendo. Por ello, era crítico que el rol del directivo se reinvente para asegurar que a pesar de las dificultades se asegure que los estudiantes logren aprendizajes de calidad. Concordamos con Bolívar (2010, citado en Freire y Miranda, 2014, p. 13) cuando afirma que, "el liderazgo pedagógico está centrado en la organización de buenas prácticas pedagógicas y en la contribución al incremento de los resultados del aprendizaje".

Los que dirigimos las instituciones educativas tenemos claro que, la forma de desarrollo de las acciones de aprendizaje se ha replanteado, pero, el contenido sigue siendo el mismo puesto que, el centro de nuestra atención educativa son los estudiantes y que todas las acciones como la movilización de recursos humanos, materiales y tecnológicos permitan que los estudiantes logren aprender.

De acuerdo a Uribe (2007), el rol de los directivos es clave para que las instituciones generen condiciones que promuevan la eficacia de la organización. Por esta razón, el liderazgo directivo es clave en este proceso de clases a distancia, pues nos enfrentamos a retos nunca antes vividos, a incertidumbre y demandas que deben ser atendidas de manera inmediata y con mucha prudencia.

Por otro lado, teniendo en cuenta que, el rol del director es gestionar la escuela para lograr aprendizajes en los estudiantes, a partir del liderazgo pedagógico que ejerce a través de un seguimiento permanente de la gestión pedagógica; por lo que, el director no puede dejar de lado el aspecto pedagógico en la gestión escolar (Freire y Miranda, 2014). En esa misma línea, "el director, como principal responsable de la gestión escolar, cumple un papel central al articular, conducir y facilitar una serie de procesos al interior de la escuela" (Freire y Miranda, 2014, p. 12).

\section{Gestión basada en logros de aprendizaje}

De acuerdo al Marco del Buen Desempeño Directivo (MBDD), el liderazgo pedagógico del equipo directivo debe garantizar las condiciones necesarias para que los estudiantes logren aprendizajes fundamentales deseables y necesarios teniendo como base una 
Congreso Internacional de Gestión Educativa, 06 y 07 de noviembre de 2020.

Pontificia Universidad Católica del Perú

organización escolar democrática, que atienda a las necesidades y el contexto de la escuela (Ministerio de Educación, 2014).

Asimismo, el MBDD explicita que, la gestión escolar debe enmarcarse en el enfoque de derechos, puesto que la gestión basada en resultados implica que se logren aprendizajes de calidad con un sentido de inclusión y equidad. De esta manera, los tres componentes que generan sinergia en la escuela y permiten a lograr los aprendizajes esperados de calidad para todos los estudiantes está basado en la gestión de los procesos pedagógicos, la convivencia democrática e intercultural y el vínculo entre la escuela, la familia y la comunidad (Ministerio de Educación, 2014).

Teniendo en cuenta que, en una gestión escolar basada en logros de aprendizaje, el rol del directivo debe estar centrada en proporcionar todas las condiciones para que los estudiantes logren aprender y que estos aprendizajes sean de calidad para asegurar que el desarrollo de competencias se encaminen a lograr el perfil de egreso de acuerdo a lo que explicita el Currículo Nacional de la Educación Básica (CNEB) "el desarrollo y logro del Perfil de egreso es el resultado de la consistente y constante acción formativa del equipo de docentes y directivos de las instituciones y programas educativos en coordinación con las familias" (Ministerio de Educación, 2016, p. 12). Por lo tanto, una gestión basada en logros de aprendizaje debe garantizar el desarrollo de todas las competencias que se explicitan en el CNEB así como lo establece el Ministerio de Educación (2016) "El Currículo Nacional de la Educación Básica orienta los aprendizajes que se deben garantizar como Estado y sociedad" (p. 6).

\section{Desarrollo de la experiencia}

La experiencia se desarrolla desde el momento en que el gobierno decreta la emergencia sanitaria y el distanciamiento social obligatorio por el incremento de infectados por la pandemia a causa del COVID - 19 y la suspensión de clases presenciales en nuestro país.

Teniendo como principio el derecho a la educación de los estudiantes y la gestión escolar centrada en logros de aprendizaje como lo explicita el Marco del Buen Desempeño Directivo (MBDD), el liderazgo pedagógico del equipo directivo debe garantizar las condiciones necesarias para que los estudiantes logren aprendizajes fundamentales deseables y necesarios teniendo como base una organización escolar democrática, que atienda a las necesidades y el contexto de la escuela (Ministerio de Educación, 2014). De esta manera, en el contexto atípico, de retos e incertidumbres, la Institución Educativa "Augusto Salazar Bondy" del distrito de Huaribamba, provincia de Tayacaja, región Huancavelica, generó espacios virtuales para la toma de decisiones oportunas para garantizar que los estudiantes participen de la estrategia "Aprendo en Casa" (EAC).

De acuerdo a Uribe (2007), el rol de los directivos es clave para que las instituciones generen condiciones que promuevan la eficacia de la organización. Por esta razón, el liderazgo directivo es clave en este proceso de clases a distancia, pues nos enfrentamos a retos nunca antes vividos, a incertidumbre y demandas que deben ser atendidas de manera inmediata y con mucha prudencia. Por ello, se generó la movilización de todos los recursos humanos de la institución y, conjuntamente con las autoridades comunales y padres de familia lograr el acceso a la educación de los estudiantes a través de la EAC. Rescatando con ello, la importancia del vínculo que debe existir entre la escuela, la

El rol del director, ¿cómo lograr el aprendizaje de los estudiantes - Gamarra Ramos, Ana Cecilia DOI: https://doi.org/10.18800/cige2020.011 
Congreso Internacional de Gestión Educativa, 06 y 07 de noviembre de 2020.

Pontificia Universidad Católica del Perú

familia y la comunidad; otorgándole un rol protagónico a cada uno de los actores involucrados con acciones de colaboración mutua y alianzas estratégicas que aporten al desarrollo común $\mathrm{y}$, por ende, se garantice que los estudiantes logren aprendizajes (Minedu, 2014).

Un aspecto central que tuvo desde el inicio la experiencia, fue garantizar el desarrollo de todas las competencias y, por ende, el involucramiento y desarrollo de todas las áreas curriculares, puesto que, como institución y de acuerdo al Ministerio de Educación (2016) "El Currículo Nacional de la Educación Básica orienta los aprendizajes que se deben garantizar como Estado y sociedad" (p. 6).

Por esta razón, en reuniones colegiadas virtuales se socializó el marco de referencia de la EAC, y resultó crítico y urgente la necesidad de que los estudiantes desarrollen todas las competencias del Currículo Nacional de la Educación Básica (CNEB), teniendo como fundamento que, los estudiantes al finalizar sus escolaridad deban desarrollar todas las competencias básicas, es así que desde la segunda semana del desarrollo de la EAC, nuestra institución cumple un horario escolar semanal a partir de la programación de la EAC, este horario contempla áreas curriculares priorizadas por la EAC y las áreas curriculares de acuerdo al plan de estudio, garantizando con ello, que los estudiantes desarrollen todas las competencias del Currículo Nacional.

Asimismo, se veló en todo momento que los estudiantes accedan a todas las condiciones críticas para asegurar su participación en las clases a distancia y sobre todo sientan que la institución está en constante evaluación y toma de decisiones de la EAC para que ellos puedan lograr aprendizajes y aseguren el desarrollo de las competencias. Partiendo por determinar que la dinámica de gestión escolar debe estar centrada en logros de aprendizaje y que para ello ocurra es necesario dinamizar todos los recursos disponibles en las instituciones educativas.

En este contexto, era necesario involucrar a este desafío al equipo directivo, personal docente y administrativo de la institución. Por lo tanto, se reorganizó y redefinió funciones de todos los que integramos la comunidad educativa, algunos aspectos a destacar fue prever el inicio de clases y con ello adecuar el currículo al contexto que se presentaba para ello, a través de la Coordinación Pedagógica se incidió en la reformulación del currículo alineado al contexto de las clases a distancia, evaluar el marco de la EAC, incidir en las características del material educativo y sobre todo con el monitoreo de la práctica pedagógica a los docentes se garantice un adecuado desarrollo de las clases a distancia para que los estudiantes logren aprendizajes

De esta manera, los docentes se convierten en los principales actores educativos para brindar oportunidades de aprendizaje, acompañar, retroalimentar, desarrollar competencias y capacidades, desarrollar los enfoques transversales, entre otras acciones para garantizar aprendizajes en los estudiantes. A este trabajo, se integra la mentoría para detectar aspectos problemáticos que tienen los estudiantes y tomar decisiones inmediatas para apoyarlos en el proceso de aprendizaje.

En este contexto de incertidumbre y con cifras alarmantes de muertes por COVID-19 se tornó crucial brindar con mayor incidencia la Tutoría y Orientación Educativa (TOE), el soporte emocional básico para asegurar la convivencia escolar, asegurar que lo estudiantes estén emocionalmente bien para garantizar el aprendizaje y por supuesto, que este soporte emocional asegure que el personal docente y administrativo se sienta emocionalmente bien para atender con pertinencia a los estudiantes y puedan desarrollar 
Congreso Internacional de Gestión Educativa, 06 y 07 de noviembre de 2020.

Pontificia Universidad Católica del Perú

mejor su trabajo. Este proceso es desarrollado por la Coordinación de Tutoría con apoyo de los docentes tutores y el psicólogo de la institución.

Por otro lado, se la conformó el Equipo Tecnológico Institucional integrado por la Coordinadora de Innovación y Soporte Tecnológico (CITS), la Docente del Aula de Innovación Pedagógica (DAIP) y el Auxiliar de Educación (AU) quienes básicamente se encargan de generar y actualizar la base de datos de conectividad de los estudiantes y sus familias, realizar alfabetización digital a los estudiantes y todo el personal de la institución, descargar y editar la transmisión de la programación de AEC tanto de la plataforma, Tv y radio; los mismos que son enviados a los docentes, estudiantes y publicarlos en la página oficial de la institución, el Facebook institucional, el canal youtube y todos los grupos de WhatsApp; entre otras funciones delegadas por la dirección.

Asimismo, el Coordinador Administrativo y de Recursos Educativos (CARE), cumple un rol fundamental en la gestión escolar, básicamente en el aspecto administrativo ya que, garantiza la asistencia y permanencia del personal docente y administrativo de la institución, asegura que todos los estudiantes cuentes con los recursos y materiales educativos dotados por el Minedu para el desarrollo de las clases a distancia, asegura la distribución oportuna y sin condicionamiento de los alimentos escolares dotados por el Programa Nacional de Alimentación Escolar (PNAE) Qali Warma para que los estudiantes consuman alimentos nutritivos en casa y puedan tener una participación activa en las clases a distancia. Asimismo, el CARE moviliza los recursos económicos del Programa Mi Mantenimiento del Minedu para garantizar mejoras en infraestructura educativa ante un inicio de clases a mediano plazo y por supuesto, garantiza la vigilancia y aseso permanente de la institución a través del personal de vigilancia y personal de servicio; entre otras funciones que son delegadas por la Dirección.

Es importante resaltar que, todos los recursos humanos, materiales, tecnológicos confluyen en garantizar la presencia de los estudiantes en las clases y, por ende, al logro de aprendizajes. Asimismo, existe un proceso permanente de evaluación de la estrategia para la toma de decisiones y estamos en un constante proceso de mejora continua para ofrecer mejores condiciones y oportunidades a nuestros estudiantes ya que, ellos son el centro de nuestro interés y son la esencia de la gestión escolar.

En la actualidad, de acuerdo a la base de datos de la I.E. Augusto Salazar Bondy (2020)

contamos con todos los estudiantes contactados a la EAC, pero no todos acceden a esta estrategia, a causa de diversos problemas que ya han sido comunicados a la Coordinación de Tutoría, los problemas encontrados son básicamente familiares por hogares disfuncionales, abandono de los padres de familia a sus hijos, escasa expectativa futura de los estudiantes por el mismo entorno familiar en el cual se desarrollan. A continuación, se muestra, algunos gráficos con respecto a lo expuesto. 


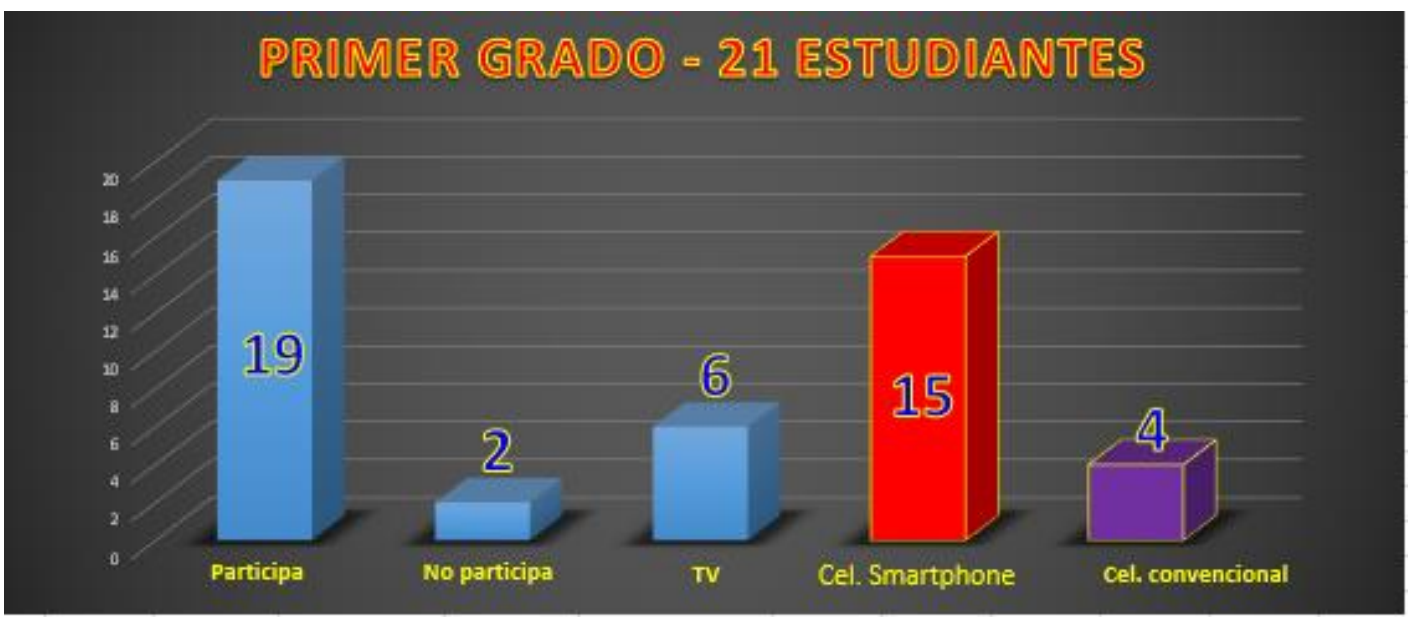

Elaboración propia.

Fuente: Base de datos I.E Augusto Salazar Bondy (2020)

Figura 1 Estudiantes del primer grado que acceden a la Estrategia Aprendo en Casa.

El siguiente gráfico muestra la cantidad de estudiantes del primer grado que han sido contactados al 100\%, de los cuales 2 no participan, representado el 10.52\%; solo 6 estudiantes, el 31,5\% tienen TV. en casa; 15 estudiantes, el 78, 94\% acceden a las clases con celular Smartphone y 4, que representa el $21,05 \%$ cuenta con celular convencional.

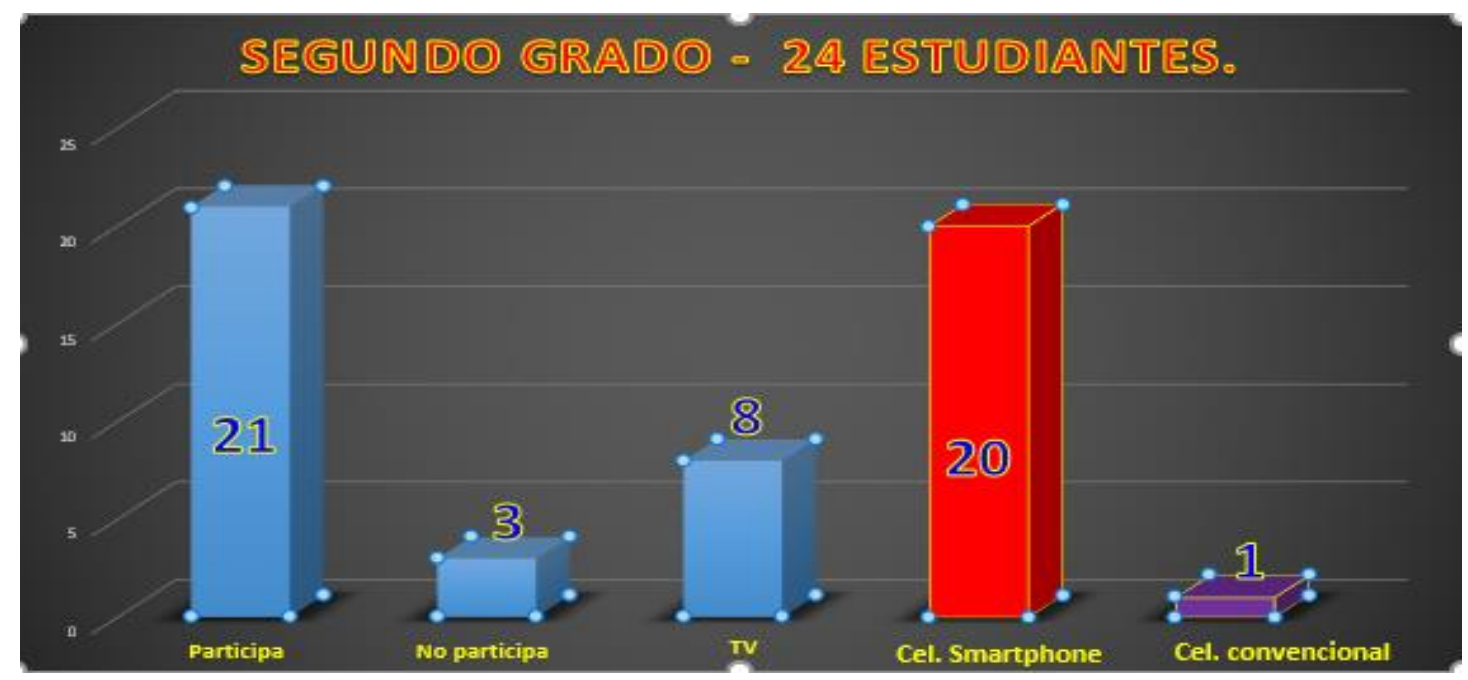

Elaboración propia.

Fuente: Base de datos I.E Augusto Salazar Bondy (2020)

Figura 2 Estudiantes del segundo grado que acceden a la Estrategia Aprendo en Casa.

La figura muestra la cantidad de estudiantes del segundo grado que han sido contactados al $100 \%$, de los cuales 3 no participan, representado el $14.28 \%$; solo 8 estudiantes que representa el 38,09\% tienen TV. en casa; 20 estudiantes, el 95,23\% acceden a las clases con celular Smartphone y 1, que representa el 4,76\% cuenta con celular convencional. 
Congreso Internacional de Gestión Educativa, 06 y 07 de noviembre de 2020.

Pontificia Universidad Católica del Perú

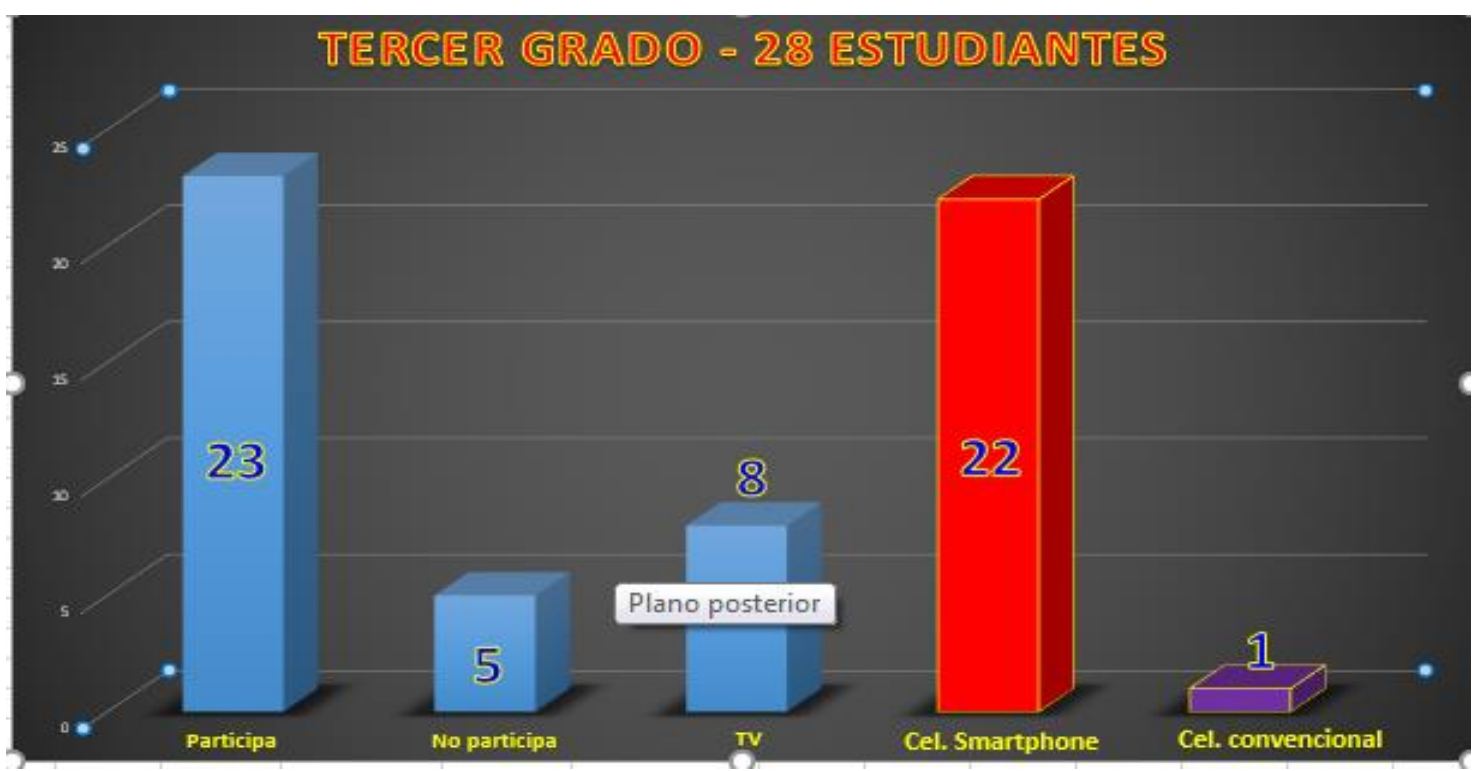

Elaboración propia.

Fuente: Base de datos I.E Augusto Salazar Bondy (2020)

Figura 3 Estudiantes del tercer grado que acceden a la Estrategia Aprendo en Casa.

La figura muestra la cantidad de estudiantes del tercer grado contactados al 100\%, de los cuales 5 no participan, representado el 21,73\%; solo 8 estudiantes que representa el 34, $78 \%$ tienen TV. en casa; 22 estudiantes, el 95,65\% acceden a las clases a distancia con celular Smartphone y 1, que representa el 4,34\% cuenta con celular convencional.

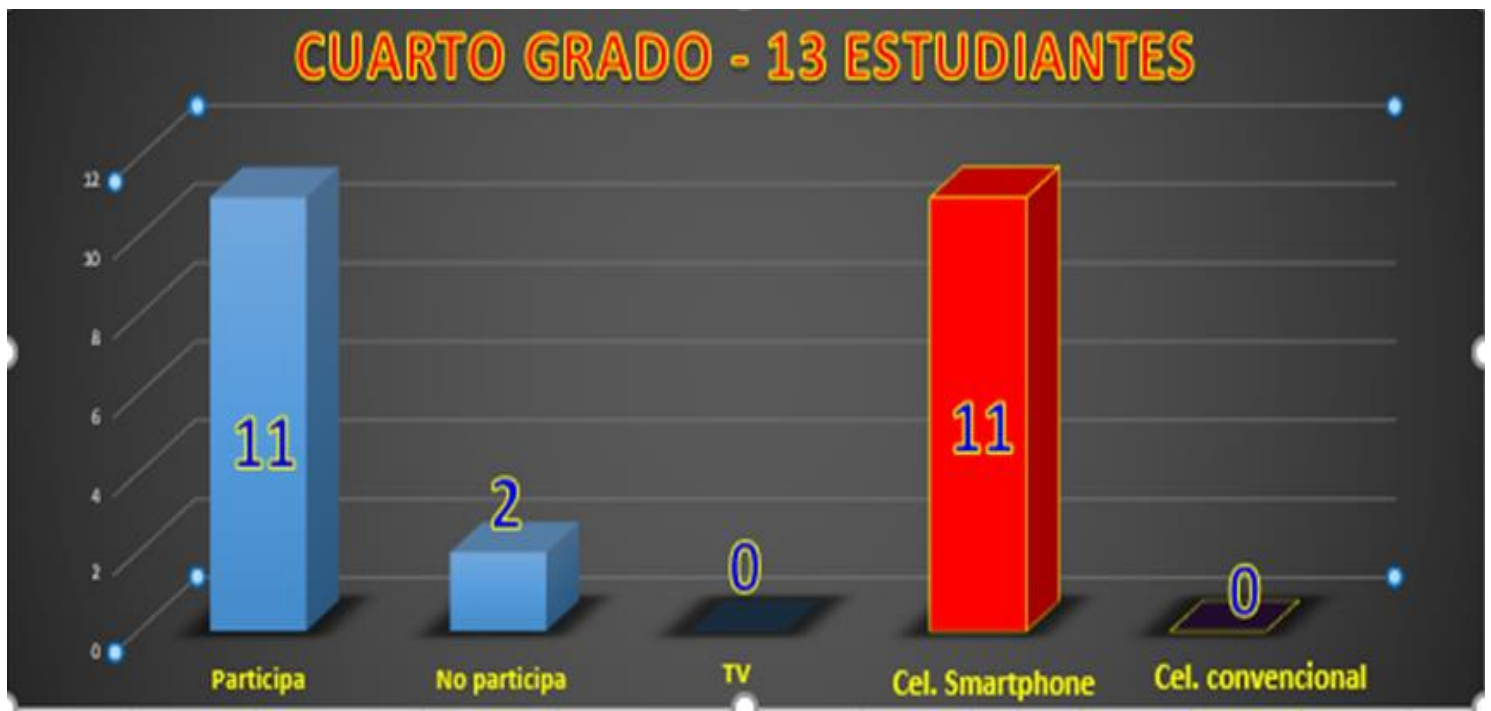

Elaboración propia.

Fuente: Base de datos I.E Augusto Salazar Bondy (2020)

Figura 4 Estudiantes del cuarto grado que acceden a la Estrategia Aprendo en Casa.

La figura muestra la cantidad de estudiantes del cuarto grado contactados al 100\%, de los cuales 2 no participan, representado el 14,38\%; de los cuales nadie tiene TV en casa, representando el $0 \% ; 11$ estudiantes, el $84,61 \%$ acceden a las clases a distancia con celular Smartphone, ningún estudiante tiene celular convencional, que representa el $0 \%$. 


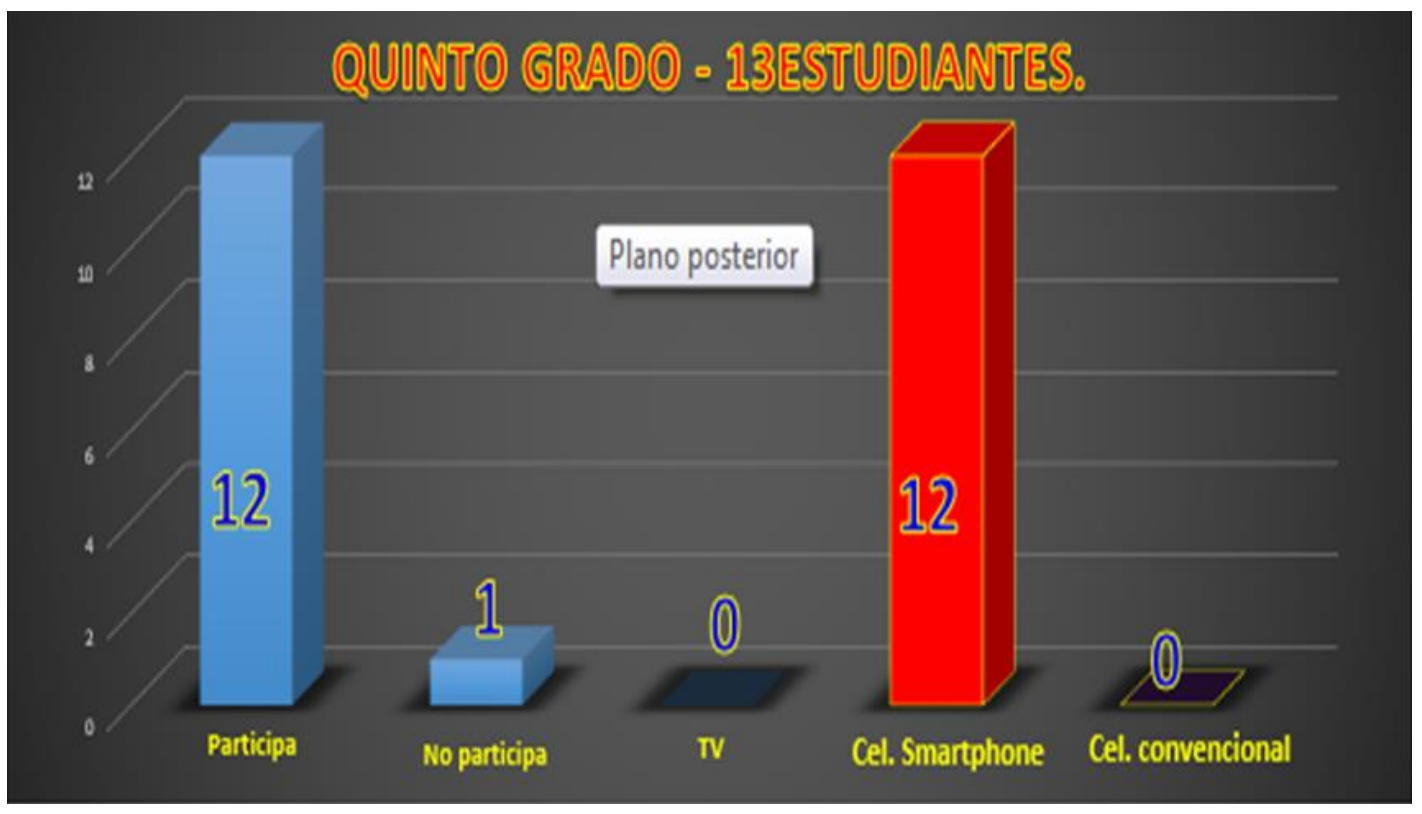

Elaboración propia.

Fuente: Base de datos I.E Augusto Salazar Bondy (2020)

Figura 5 Estudiantes del quinto grado que acceden a la Estrategia Aprendo en Casa.

La figura muestra la cantidad de estudiantes del quinto grado que han sido contactados al 100\%, de los cuales 1 no participa, representado el 7,6\%; de los cuales nadie tiene TV en casa, representando el 0\%; 12 estudiantes, el 92,3\% acceden a las clases a distancia con celular Smartphone, ningún estudiante tiene celular convencional, que representa el $0 \%$.

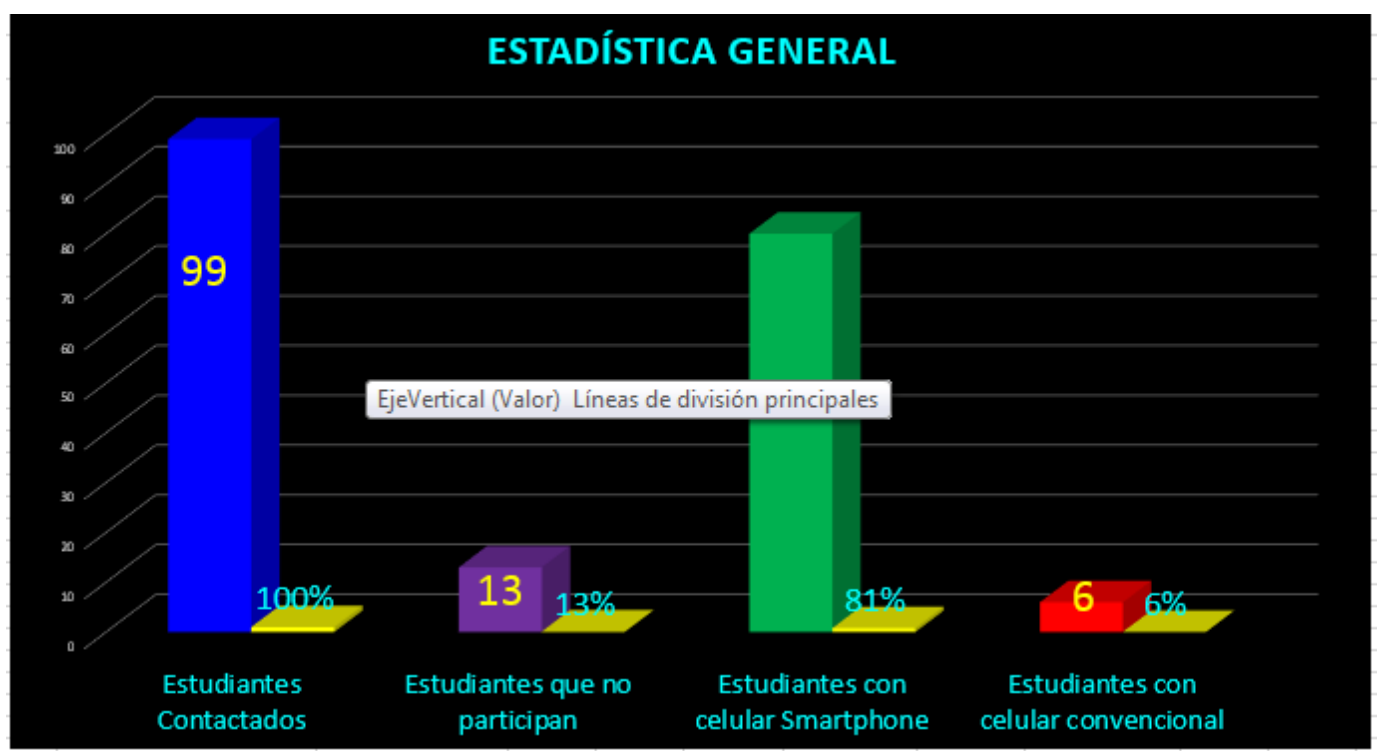

Elaboración propia.

Fuente: Base de datos I.E Augusto Salazar Bondy (2020)

Figura 6 Acceso institucional a la estrategia aprendo en casa 
Congreso Internacional de Gestión Educativa, 06 y 07 de noviembre de 2020.

Pontificia Universidad Católica del Perú

La figura nos muestra la cantidad de estudiantes que al mes setiembre han sido contactados al $100 \%$, de los cuales el $13 \%$ de los estudiantes que, a pesar de haber sido contactados no acceden a la EAC; el $81 \%$ acceden a las clases con celular Smartphone; el $6 \%$ acceden a las clases con celular convencional.

De acuerdo a lo explicitado, nos queda como reto institucional reinsertar a los estudiantes que no participan en la EAC y con ello, garantizar el derecho a la educación y que los docentes y todo el personal de la institución ayuden a nuestros estudiantes a encaminarse y generar todas las condiciones y oportunidades para ayudarlos en esta tarea de desarrollo de competencia y por ende, que ellos no pierdan la oportunidad de aprender que es la base para su desarrollo personal.

\section{Lecciones prendidas}

El rol del director debe asegurar los logros de aprendizajes de los estudiantes.

El rol del director debe estar centrado en liderar la gestión pedagógica y lograr aprendizajes de calidad.

El director debe asegurar instituciones educativas con sentido de urgencia, integradoras, inclusivas y resilientes ante futuros contextos desafiantes.

El director debe liderar la gestión escolar, repensar y reimaginar de manera permanente la tarea educativa.

El director debe ser retador y tomar decisiones de manera oportuna para dinamizar todos los recursos en beneficio de los logros de aprendizaje.

\section{Agradecimientos}

Agradecimiento especial a toda la comunidad educativa augustina del distrito de Huaribamba, provincia de Tayacaja de la región Huancavelica por el compromiso, responsabilidad y el trabajo en equipo demostrado en este proceso de desarrollo de la estrategia "Aprendo en Casa". Nada sería posible si no generamos sinergia entre todos los que integramos la institución y que juntos podemos garantizar que los estudiantes logren aprendizajes de calidad.

\section{Referencias bibliográficas}

Freire, S., \& Miranda, A. (2014). El rol del director en la escuela: el liderazgo pedagógico y su incidencia sobre el rendimiento académico. Recuperado de http://repositorio.grade.org.pe/bitstream/handle/GRADE/297/AI17.pdf?sequence $=1 \&$ is

Ministerio de Educación (2014). Marco del buen desempeño directivo. Directivos construyendo escuela. Recuperado de http://www.minedu.gob.pe/n/xtras/marco_buen_desempeno_directivo.pdf

Ministerio de Educación (2016). Currículo de la Educación Básica Regular. Recuperado de http://www.minedu.gob.pe/curriculo/pdf/curriculo-nacional-de-la-educacionbasica.pdf 
Congreso Internacional de Gestión Educativa, 06 y 07 de noviembre de 2020.

Pontificia Universidad Católica del Perú

Talanquer, Vicente. (2015). La importancia de la evaluación formativa. Educación química, 26(3), 177-179. https://doi.org/10.1016/j.eq.2015.05.001

Uribe, M. (2007). Liderazgo y competencias directivas para la eficacia escolar: experiencia de modelo escolar de fundación Chile. Revista Iberoamericana sobre Calidad, Eficacia y Cambio en Educación, 5(5), 149-156. Recuperado de https://www.redalyc.org/pdf/551/55121025022.pdf 